\title{
Anionic polysaccharides modified filter papers for rapid isolation of extracellular vesicles from diverse samples in a simple bind-wash-elute manner
}

\author{
Shanglin $\mathrm{Li}^{1}$, Qiang $\mathrm{Liu}^{2}$, Zhi Geng ${ }^{1}$, Kaiyi $\mathrm{Li}^{1}$, Tian Zhao ${ }^{1}$, and Peng Liu ${ }^{1, *}$
}

${ }^{1}$ Department of Biomedical Engineering, School of Medicine, Tsinghua University, Beijing, 100084, China.
${ }^{2}$ Beijing Haidian Hospital, Beijing, 100080, China.

* Corresponding authors: Peng Liu, Department of Biomedical Engineering, School of Medicine, Tsinghua University, Haidian District, Beijing, 100084, China. Phone: +86-10-62798732, fax: +86-10-62798732, email: pliu@tsinghua.edu.cn

\section{Supporting information}

\section{Table of contents}

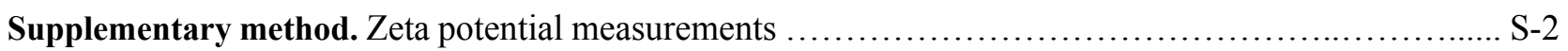

Supplementary method. Enzyme-linked immunosorbent assay (ELISA) for EV quantification .............. S-2

Supplementary method. Confocal imaging of cellular uptakes of isolated EVs ........................... S-2

Supplementary method. Immunoblotting (Western Blotting) ...........................................

2

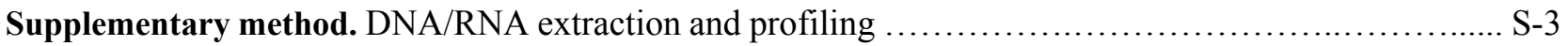

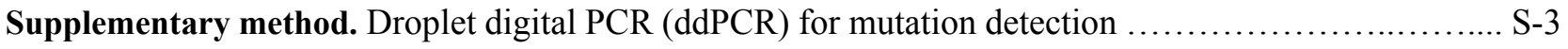

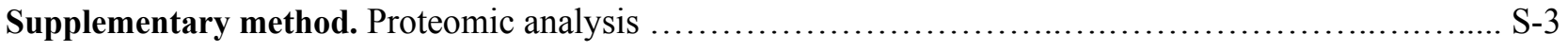

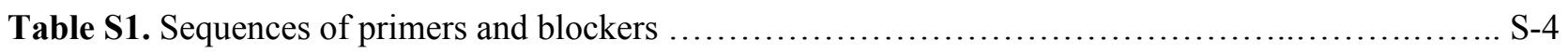

Table S2. AppiEV parameters for diverse biological fluids ..................................... S-6

Figure S1. High-resolution X-ray photoelectron spectroscopic profiles of modified glass fibers ................ S-7

Figure S2. Zeta potential distributions of standardized EVs with Alg, CS, and HS in buffer solution ........... S8

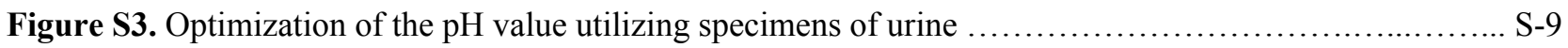

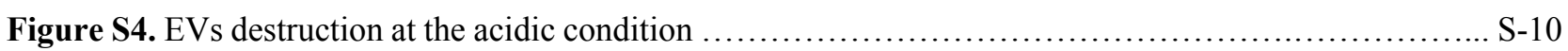

Figure S5. Transmission electron microscopy (TEM) images of the EVs isolated by Alg-AppiEVs, CS-AppiEVs, HS-ApiEVs, and UC from plasma, urine, and cell culture supernatants .....................................

Figure S6. Size distributions of EVs products isolated by AppiEV at different $\mathrm{pH}$ values .................... S-12 
Figure S7. Transmission electron microscopy (TEM) images of the isolated EVs

S-13

Figure S8. Size and quality of RNA extracted from EV products 


\section{Supplementary materials and methods}

Zeta potential measurements. To verify the feasibility of the electrostatic model, the standardized EVs were isolated by $\mathrm{UC}$ at $100000 \mathrm{~g}$ for $70 \mathrm{~min}$ from decellularized cell supernatants and resuspended into phosphate buffer saline (PBS). The standardized EV samples were freshly diluted by 100 volume of NaAc buffer at $\mathrm{pH} 4.0,5.0$, and 6.0 respectively, and immediately loaded onto the Zetasizer Nano ZS instrument (Malvern Instruments, Malvern, United Kingdom) for zeta potential analysis. To verify the feasibility of anionic polysaccharides for EV capture, the standardized EVs were dispersed into NaAc buffer at $\mathrm{pH} 4.0$ with $82.7-\mu \mathrm{g} / \mathrm{ml} \mathrm{Alg,} \mathrm{104.2-} \mu \mathrm{g} / \mathrm{ml} \mathrm{HS}$, and 138.4$\mu \mathrm{g} / \mathrm{ml} \mathrm{CS}$, respectively. After a 30-min incubation, the EVs in anionic polysaccharides solution was isolated again by UC at $100000 \mathrm{~g}$ for $70 \mathrm{~min}$ and resuspended into PBS. Finally, the EV solution was freshly diluted by 100volume NaAc buffer at pH 4.0 and analyzed by the Zetasizer Nano ZS instrument.

Enzyme-linked immunosorbent assay (ELISA) for EV quantification. To improve the isolation efficiency of AppiEV, the $\mathrm{pH}$ value and betaine in the capture buffer and the surface ion types of modified filter paper were optimized by ELISA for CD63 kit (ELISA Kit for CD63 antigen, Wuhan EIAab Science Inc., China) using the pretreated cell culture supernatant. The isolation efficiency of these three types of anionic polysaccharides modified GFs were measured by ELISA using a standardized solution, which was prepared by adding EVs, into DMEM with 100 units $/ \mathrm{mL}$ penicillin and $100 \mu \mathrm{g} / \mathrm{mL}$ streptomycin. The standardized EV solution was prepared by distributing EVs isolated from cell culture supernatant by UC at $100000 \mathrm{~g}$ for $70 \mathrm{~min}$ into PBS. The concentrations of CD63, Hsp90 alpha, and PD-L1 in elute were measured using commercial ELISA kits all from FEiMo (FEiMo bio life science Inc., Beijing, China) following the manufacturer's protocols.

Confocal imaging of cellular uptakes of isolated EVs. To verify the functional activity of isolated EVs, we labeled the isolated EVs with 1:10,000 dilution of PKH67 (PKH67 Green Fluorescent Cell Linker Mini Kit, SigmaAldrich), a fluorescent dye for cell membrane labeling. Then, the labeled EVs were resuspended in $10 \mathrm{~mL}$ of DMEM containing 1\% (v/v) penicillin and streptomycin solution and incubated with PC9 cells in 35-mm confocal dishes. After a 5-minute incubation, the cells were washed three times with PBS and fixed with 4\% paraformaldehyde for $10 \mathrm{~min}$. Finally, confocal images were obtained with a $63 \mathrm{X}$ objective on an LSM 710 confocal laser scanning microscope (Zeiss, Oberkochen, Germany).

Immunoblotting (Western Blotting). EV products were lysed by 1\% (v/v) NP40 and the total proteins were quantified by BCA protein assay kit (Tiangen Biotech, Beijing, China) according to its protocol. Each of 10- $\mu \mathrm{g}$ 
total proteins was resolved by SDS/PAGE and transferred onto PVDF membranes. Membranes were blocked for $1 \mathrm{~h}$ with $5 \%$ BSA in Tris-buffered saline containing $0.1 \%$ Tween 20 and incubated overnight at $4{ }^{\circ} \mathrm{C}$ with antiTsg101 antibody, anti-Hsp70 antibody, anti-CD81 antibody, anti-Apo B antibody, and anti-calnexin antibody all from Abcam (Abcam, Cambridge, UK). Membranes were washed 30 min with Tris-buffered saline containing $0.1 \%$ Tween 20, incubated for $1 \mathrm{~h}$ with goat anti-rabbit IgG antibody conjugated to horseradish peroxidase, and developed using chemiluminescent substrates.

DNA/RNA extraction and profiling. RNA was extracted from EVs using the miRNeasy mini kit (Qiagen, Hilden, Germany) according to the standard protocol. The quality and the size of RNA were analyzed using the Agilent RNA 21000 Pico kit (Agilent Technologies, Santa Clara, CA) on an Agilent 2100 Bioanalyzer $^{\circledR}$ (Agilent Technologies, Santa Clara, CA). Similarly, DNA was extracted from EVs using the DNA mini kit (Qiagen, Hilden, Germany). The quality and the size of DNA were analyzed using the Agilent high sensitivity DNA kit on the Agilent 2100 Bioanalyzer ${ }^{\circledR}$.

Droplet digital PCR (ddPCR) for mutation detection. For the mutation detection of EGFR p.L858R, after isolating EVs from $1 \mathrm{~mL}$ plasma samples, the isolated EVs were lysed by protein K (Solarbio, Beijing, China) at $55^{\circ} \mathrm{C}$ for $10 \mathrm{~min}$ followed by $95^{\circ} \mathrm{C}$ for $5 \mathrm{~min}$ to inactivate the protein $\mathrm{K}$. After the lysis of EVs, the specific reverse transcription of $E G F R$ gene was performed in a $20-\mu \mathrm{L}$ reaction system contained $1-\mu \mathrm{L}$ RT enzyme (ReverTra Ace ${ }^{\circledR}$ qPCR RT Kit, TOYOBO, Japan), 1X RT buffer (5X, ReverTra Ace ${ }^{\circledR}$ qPCR RT Kit), 500-nM each of EGFR primers (listed in SI Table 1), and $14-\mu \mathrm{L}$ lysed product solution at $37^{\circ} \mathrm{C}$ for 15 min followed with $98^{\circ} \mathrm{C}$ for 5 min to inactivate the enzyme. Finally, the $10-\mu \mathrm{L}$ RT product was analyzed by the ddPCR using human EGFR p.L858R assay (Apexbio, Beijing, China) on a Naica ${ }^{\mathrm{TM}}$ Crystal Digital PCR System (Stilla Technologies, Paris, France).

Proteomic analysis. Mass spectrometry (MS) analysis of isolated EVs performed using $20-\mu \mathrm{g}$ proteins. Proteins were separated by $10 \%$ SDS-PAGE electrophoresis and stained by coomassie brilliant blue fast staining solution (Solarbio life sciences, Beijing, China). Then each of the protein bands was cut and transferred into tubes. After washing twice with ultrapure water, the protein gels were destained using a destaining solution $\left(25-\mathrm{mM} \mathrm{NH}_{4} \mathrm{HCO}_{3}\right.$, $50 \%$ acetonitrile) at $37^{\circ} \mathrm{C}$ for $1 \mathrm{~h}$. After rinsed with acetonitrile three times, dried at rotary vacuum drier for $5 \mathrm{~min}$. Double volume 25 -mM DTT solution was added into the tube and incubated at $55^{\circ} \mathrm{C}$ for $45 \mathrm{~min}$. After removing the DTT solution, the gel was rinsed three times with acetonitrile and dried at a rotary vacuum drier for 5 min. Double volume 50-mM IAA solution was added into the tube and incubated in the dark for $30 \mathrm{~min}$. After removing 
the IAA (indoleacetic acid) solution, the gel was rinsed three times with acetonitrile and dried at a rotary vacuum drier for $5 \mathrm{~min}$. The diluted pancreatin solution $(10-\mu \mathrm{g}$ diluted by $790-\mu \mathrm{L}$ pancreatin dilution buffer) was added into the tube and incubated at $37^{\circ} \mathrm{C}$ for $12 \sim 16 \mathrm{~h}$. To terminate the reaction, $20-\mu \mathrm{L}$ trifluoroacetic acid was added into the tube and then removed supernatant. The extraction liquid (50\% acetonitrile, $0.1 \%$ formic acid) was injected into a tube and incubated at $37^{\circ} \mathrm{C}$ for $45 \mathrm{~min}$ and repeat three times. The supernatant was collected and dried at a rotary vacuum drier and dissolved by $0.1 \%$ formic acid solution for LC-MS/MS (SYNapt G2, Thermo Fisher Scientific, Massachusetts). Each of the samples was separated by liquid chromatography (Thermo-Dionex Ultimate 3000, Thermo Scientific). Peptides were separated using a $75-\mu \mathrm{m}$-inner-diameter C18 column (5 $\mu \mathrm{m}$ beads, Thermo Scientific) at a flow rate of $0.3 \mu 1 / \mathrm{min}$, with a mobile phase A ( $0.1 \%$ formic acid) and a mobile phase B $(0.1 \%$ formic acid in acetonitrile). All LC-MS/MS experiments were performed in data-dependent mode using Xcalibur 4.0.27.10 software. Precursor mass spectra were recorded in a 300 1,500 m/z mass range. Data were extracted and searched Uniprot complete Human proteome databases using Proteome Discoverer and the genes annotated using Gene Ontology database. We acknowledge the support from Xiaolin Tian and her colleagues in Tsinghua University Technology Center of Protein Research. 
Supplementary table

Table S1: Sequences of primers and blockers

\begin{tabular}{c|lc}
\hline Experiments & \multicolumn{2}{|c}{ Name } \\
\hline \multirow{2}{*}{ EGFR } & EGFR-F & 5'-GCTCCCAGTACCTGCTCAAC-3' \\
\cline { 2 - 3 } & EGFR-R & 5'-TGGCTTGGATCCAAAGGTCA-3' \\
\hline \multirow{4}{*}{ EGFR p.T790M } & T790M-F & 5'-ACCATGCGAAGCCACACTGACG-3' \\
\cline { 2 - 3 } & T790M-R & 5'-GCCGAAGGGCATGAGCTGGA-3' \\
\cline { 2 - 3 } & T790M-blocker & 5'-CATGAGCTGC/LNA_G/TGATGAGCTGCAC- \\
& & GGTGGAG/C3 spacer/-3' \\
\hline \multirow{2}{*}{ U6 } & U6-F & 5'-CTCGCTTCGGCAGCACA-3' \\
\cline { 2 - 3 } & U6-R & 5'-AACGCTTCACGAATTTGCGT-3' \\
\hline
\end{tabular}


Table S2. AppiEV parameters for diverse biological fluids

\begin{tabular}{cccc}
\hline & Cell supernatant & Plasma/serum & Urine \\
\hline Diameter of filter paper $(\mathrm{mm})$ & $8,11,24$ & $8,11,24$ & 11,24 \\
Maximum volume of samples $(\mathrm{mL})$ & 10 & 5 & 20 \\
Minimum volume of eluate $(\mu \mathrm{L})$ & $30,50,100$ & $30,50,100$ & 50,100 \\
Total time $(\mathrm{min})$ & 50 & 65 & 50 \\
\hline
\end{tabular}




\section{Supplementary figures}

A

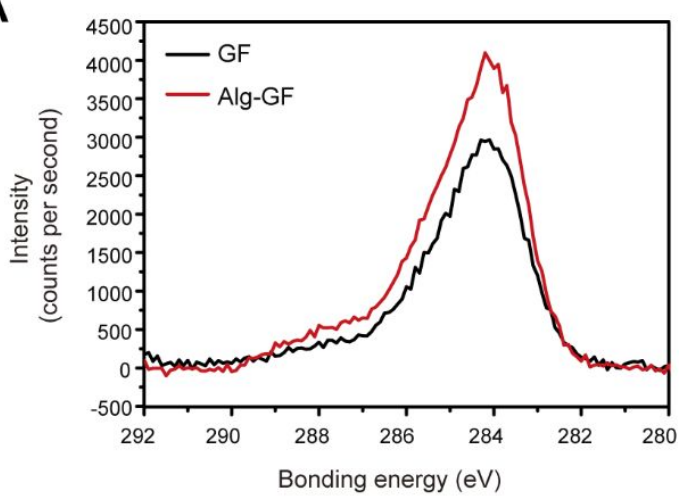

B

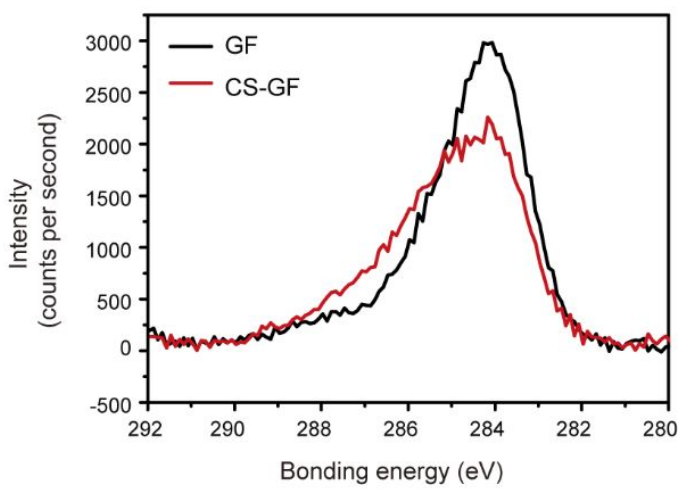

C

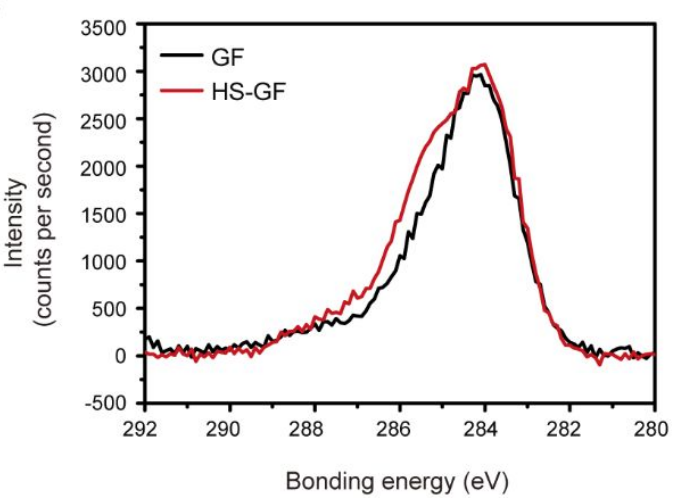

Figure S1. High-resolution X-ray photoelectron spectroscopic (XPS) profiles of the C1s core level of Alg (A), CS (B), and HS (C)-modified glass fibers. 


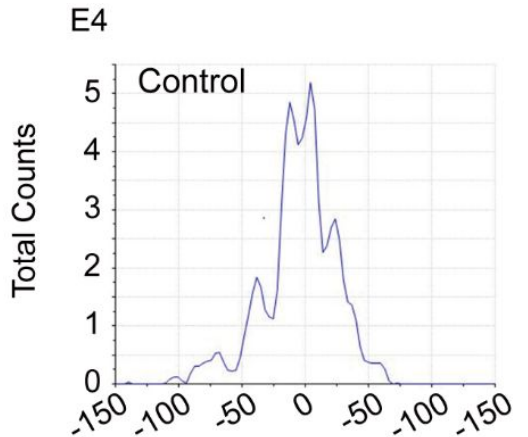

Zeta potential $(\mathrm{mV})$

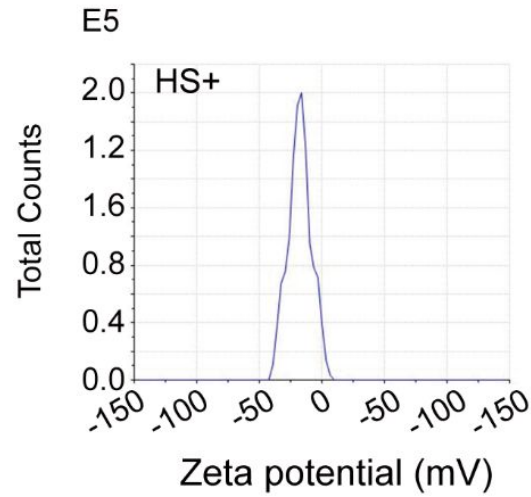

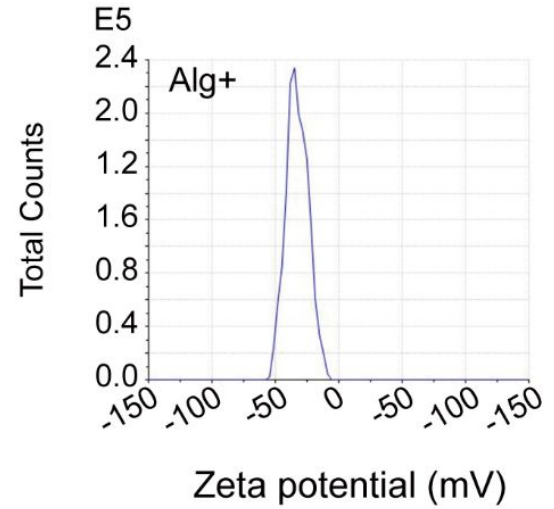

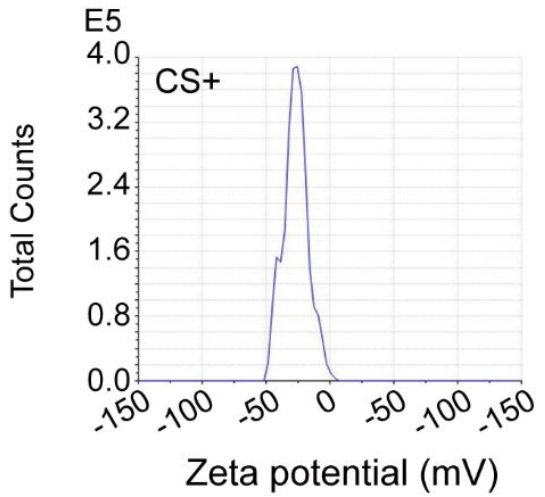

Figure S2. Zeta potential distributions of standardized EVs at pH 4.0 with Alg, CS, and HS in buffer solution. 


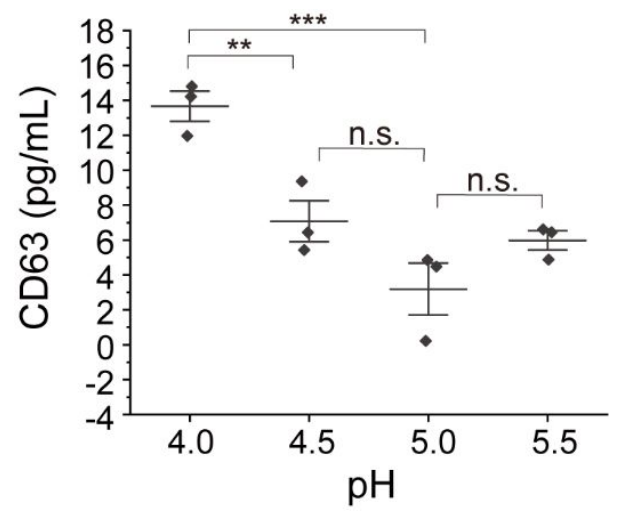

Figure S3. Optimization of the $\mathrm{pH}$ value utilizing specimens of urine. ${ }^{* * *}, \mathrm{P}<0.01$; **, $\mathrm{P}<0.05$; n.s., no significance. 
A

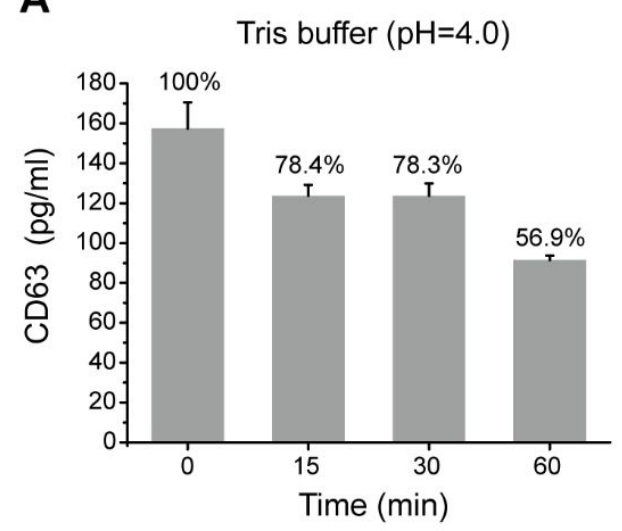

C

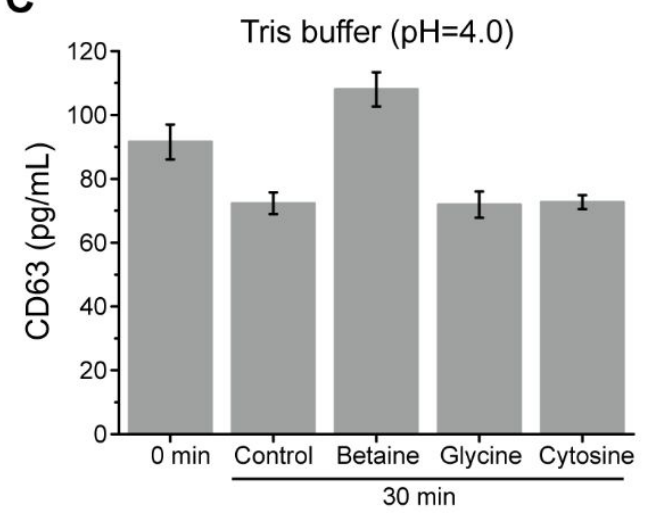

B

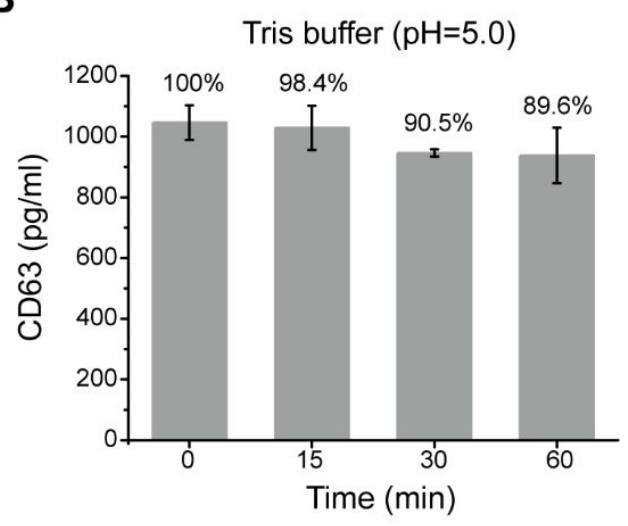

D

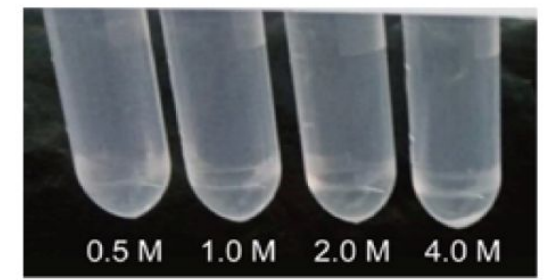

Figure S4. EVs destruction at the acidic condition. The detectable CD63 concentrations in standardized EVs solution after incubating in a Tris- $\mathrm{HCl}$ buffer at $\mathrm{pH} 4.0$ (A) and pH 5.0 (B) analyzed by ELISA. (C) The detectable CD63 concentration in standardized EVs solution after incubating in Tris- $\mathrm{HCl}$ buffer with betaine, glycine, and cytosine at $\mathrm{pH} 4.0$ for $30 \mathrm{~min}$. (D) Dissolution of EDTA2Na salt at high concentration of betaine. 


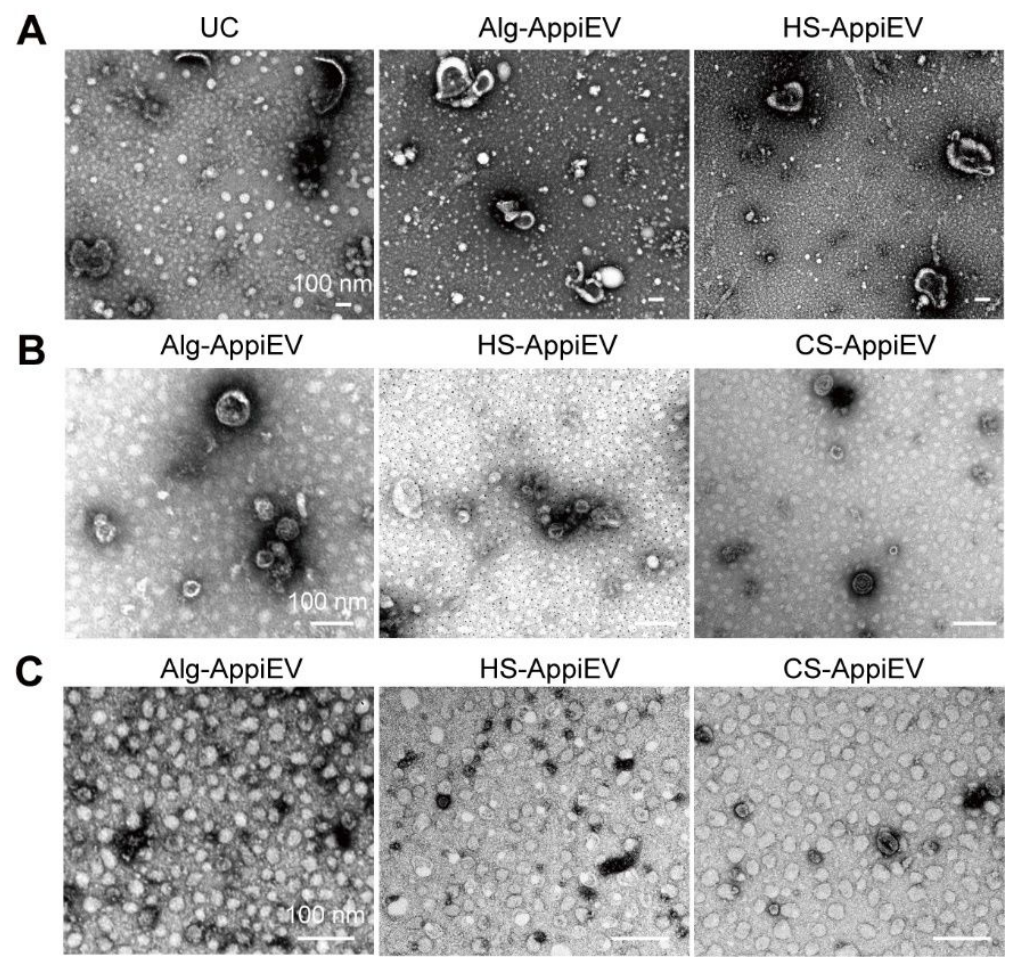

Figure S5. Transmission electron microscopy (TEM) images of the EVs isolated by Alg-AppiEVs, CS-AppiEVs, HS-ApiEVs, and UC from plasma (A), urine (B), and cell culture supernatants (C). 
A
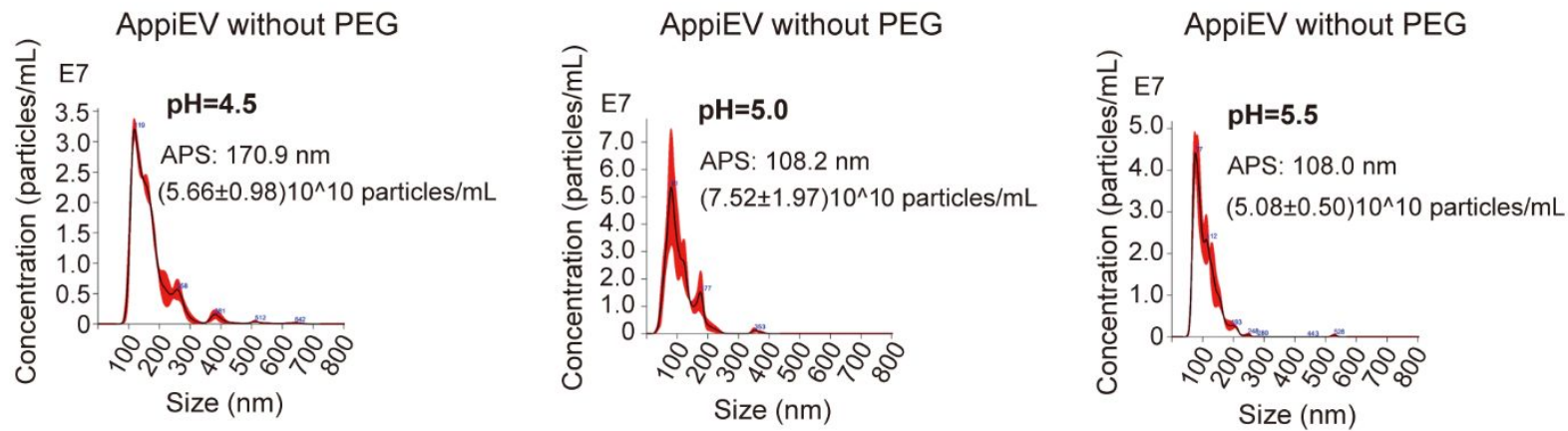

B
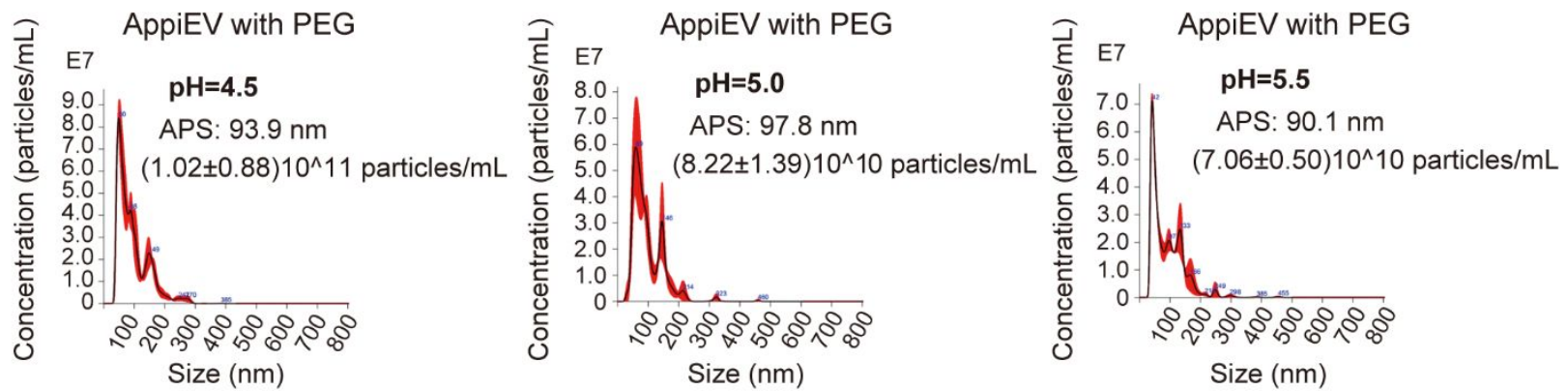

Figure S6. Size distributions of EVs products isolated by AppiEV at different $\mathrm{pH}$ values. Size distributions of EVs products isolated by AppiEV with (A) and without (B) PEG at $\mathrm{pH} 4.5,5.0$, and 5.5 were analyzed by NTA. 

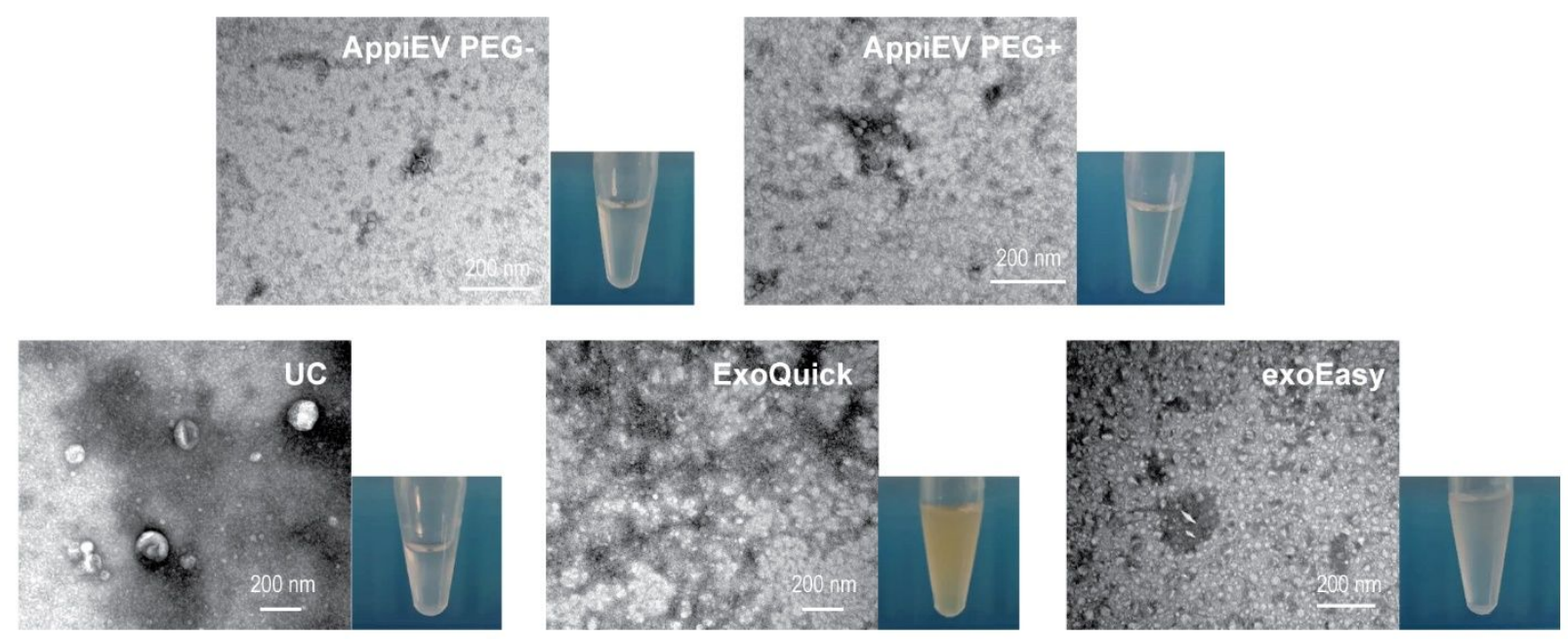

Figure S7. Transmission electron microscopy (TEM) images of the EVs isolated by AppiEV PEG-, AppiEV PEG+, UC, ExoQuick, and exoEasy from $0.5-\mu$ L plasma specimens. 

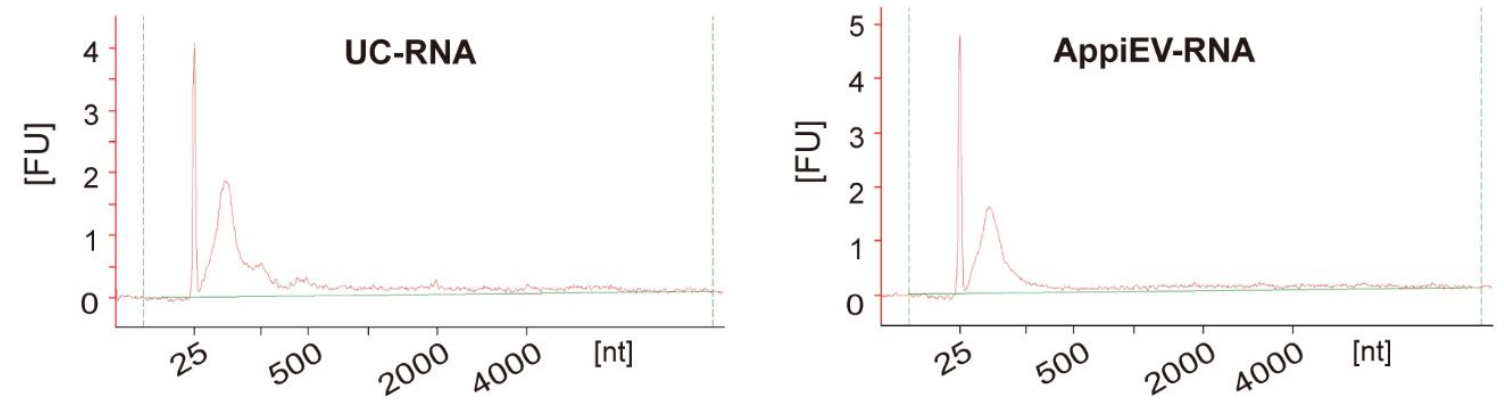

Figure S8. Size and quality of RNA extracted from EV products isolated by UC and AppiEV from the same plasma sample. 